\title{
The finite element implementation of 3D fractional viscoelastic constitutive models
}

\author{
Gioacchino Alotta $^{\mathrm{a}}$, Olga Barrera ${ }^{\mathrm{b}, \mathrm{c}, *}$, Alan Cocks ${ }^{\mathrm{c}}$, Mario Di Paola ${ }^{\mathrm{d}}$ \\ a Bio/NanoMechanics for Medical Sciences Laboratory, ATeN-Center, Universitá degli studi di Palermo, Palermo, Italy \\ ${ }^{\mathrm{b}}$ School of Engineering Computing and Mathematics, Oxford Brookes University, Oxford, UK \\ ${ }^{\mathrm{c}}$ Department of Engineering Science, University of Oxford, Oxford, UK \\ d Dipartimento di Ingegneria Civile, Ambientale, Aerospaziale, dei Materiali (DICAM), Universitá degli Studi di Palermo, Palermo, Italy
}

\section{A R T I C L E I N F O}

\section{Keywords:}

Fractional viscoelasticity

3D constitutive models

Creep

Relaxation

Numerical modelling

\begin{abstract}
A B S T R A C T
The aim of this paper is to present the implementation of 3D fractional viscoelastic constitutive theory presented in Alotta et al., 2016 [1]. Fractional viscoelastic models exactly reproduce the time dependent behaviour of real viscoelastic materials which exhibit a long "fading memory". From an implementation point of view, this feature implies storing the stress/strain history throughout the simulations which may require a large amount of memory. We propose here a number of strategies to effectively limit the memory required. The form of the constitutive equations are summarized and the finite element implementation in a Newton-Raphson integration scheme is described in detail. The expressions that are needed to be coded in user-defined material subroutines for quasi static and dynamic implicit and explicit analysis (UMAT and VUMAT) in the commercial finite element software ABAQUS are readily provided. In order to demonstrate the accuracy of the numerical implementation we report a number of benchmark problems validated against analytical results. We have also analysed the behaviour of a viscoelastic plate with a hole in order to show the efficiency of these types of models. The source codes for the UMAT and VUMAT are provided as online supplements to this paper.
\end{abstract}

\section{Introduction}

In the last decade the use of fractional viscoelastic models has gained interest among researchers as they are capable of accurately represent both creep and relaxation behaviour of viscoelastic materials and the effects of "fading" memory captured experimentally. It has been widely shown that, during a creep/relaxation test, the stress/strain response of viscoleastic materials is characterized by a power law with respect to time; examples are polymers, biological tissues, asphalt mixtures, soils ([2-6]) among others. A power-law in the creep and relaxation responses leads to fractional viscoelastic constitutive models which are characterized by the presence of derivatives and integrals of noninteger order (see Refs. [7,8]). The most attractive aspect of using fractional operators in the viscoelastic constitutive laws is that the stress/displacement response depends on the previous stress/strain history, which allows the long "fading" memory of the material to be taken into account. Another advantage of fractional viscoelastic models is that they are defined by a small number of parameters compared to classical integer order viscoelastic models. Numerous studies have been devoted to theoretical aspects of $1 \mathrm{D}$ fractional constitutive laws $([3,9-14])$ as well as experimental aspects and parameter characterization ([15-20]) of the constitutive behavior and also application to beam models subjected to both deterministic $([21,22])$ and stochastic ([23-25]) conditions. The influence of temperature on the response of fractional viscoelastic models has also been investigated ([26,27]). Some numerical implementation of 1D fractional constitutive laws in finite element codes has been presented (see for example [28]).

3D formulations of fractional viscoelastic models have been proposed and studied (see for example [1,29-32]). In order to be able to use these models to represent the behaviour of real-life engineering components with complex shapes, it is necessary to perform the implementation of these constitutive models into finite element software. To the author's knowledge the implementation of 3D formulations of fractional viscoelastic models in a finite element context is lacking. Indeed, to the best of the authors' knowledge only in Ref. [33] an effort was made to implement fractional viscoelasticity in a finite element code. However, only the fractional standard linear solid (FSLS) model was considered in the paper [33], while many researchers of

\footnotetext{
* Corresponding author. School of Engineering Computing and Mathematics, Oxford Brookes University, Oxford, UK.

E-mail addresses: gioacchino.alotta@unipa.it (G. Alotta), obarrera@brookes.ac.uk (O. Barrera), alan.cocks@eng.ox.ac.uk (A. Cocks), mario.dipaola@unipa.it (M. Di Paola).
} 
the field use also other fractional viscoelastic model such as the springpot, the fractional Kelvin-Voigt (FKV) model and the fractional Maxwell (FM) model. Hence, the aim of this paper is the implementation of the most used three dimensional fractional viscoelastic constitutive laws in finite element (FE) codes. In particular, we fully cover the details of the implementation of these models in user-defined material subroutines in the commercial finite element software ABAQUS. In our opinion, the numerical implementation of a new fractional viscoelastic theory using the finite element method is often a laborious task especially for researchers new to this area. Here we clearly show the expression of the constitutive tangent tensor that needs to be implemented in the UMAT routine; the implementation is straightforward also for researchers and engineers that have not specific knowledge of fractional calculus. The details of numerical procedures and related expressions that need to be implemented in user defined routines are not extensively published in the literature. Recently, there has been an interest in the dissemination of new computational procedures through publishing research papers addressing all of the aspects related to their implementation. For instance Chester et al. [34] recently presented the implementation of a coupled diffusion mechanics model for elastomeric gels as a user-defined element (UEL) subroutine in ABAQUS. Furthermore, the implementation of a coupled mechanics-diffusion theory in a user defined material routine (UMATHT) in ABAQUS has been presented by Barrera et al. [35] in order to study hydrogen embrittlement mechanisms of steels. Also a cohesive finite element as a UEL subroutine in ABAQUS has also been published by Park and Paulino [36]. Here we show that these 3D fractional viscoelastic models can be easily implemented numerically in a finite element context by using the discretized version of fractional derivatives provided by Grünwald-Letnikov [7]. In this paper we also include the details of computational tools used to access the strain (and/or the stress) history and the possible strategies to reduce the amount of memory required to run analysis of large FE models. This issue has not been discussed elsewhere. The source codes for implicit and explicit analysis of the 3D fractional Kelvin Voigt model are reported as an online supplement to this paper.

The paper is organized as follows: firstly the three-dimensional springpot model is summarized (this is also discussed extensively in Ref. [1]) and then its implementation is described; second, the other fractional viscoelastic models are introduced and their implementation is presented. We then discuss possible solutions to limit the memory required to run large simulations. Finally, comparisons with some benchmark problems are presented in order to show the accuracy of the routines and the possibility to reproduce a wide range of different behaviours.

\section{3D fractional constitutive law}

It is well known that a viscoelastic material can be characterized, for one dimensional problems, by its Relaxation and Creep functions $R(t)$ and $C(t)$ respectively. These functions describe the behaviour of the material when a constant strain and a constant stress are applied, respectively.

Experimental tests on real viscoelastic materials, such as polymers, asphalt mixtures, biological tissues, have shown that creep and relaxation are well fitted by power laws of real order rather than exponential functions. In the simplest case in which only one component of the stress is present (hydrostatic or tangential stress), and the creep/relaxation behaviour is well fitted by pure power laws, the relaxation function $R(t)$ and the creep function $C(t)$ are given as [1]:

$R(t)=\frac{C_{\rho} t^{-\rho}}{\Gamma(1-\rho)} ; \quad C(t)=\frac{t^{\rho}}{C_{\rho} \Gamma(1+\rho)}$

where $\Gamma(\cdot)$ is the Euler gamma function, $\rho$ is a real number $0 \leq \rho \leq 1$ and $C_{\rho}$ is a material parameter evaluated by fitting creep or relaxation experimental curves.

In the frame of linear viscoelasticity, the Boltzmann superposition principle allows us to obtain the response of a material when the imposed stress $s(t)$ or strain history $e(t)$ is not constant and can be expressed in two forms:

$s(t)=\int_{0}^{t} R(t-\tau) \dot{e}(\tau) d \tau$

$e(t)=\int_{0}^{t} C(t-\tau) \dot{s}(\tau) d \tau$

These integrals are often labelled as "hereditary" integrals, because the actual value of $s(t)$ (or $e(t)$ ) depends on the entire previous history of $e(t)$ (or $s(t))$. Eqs. (2a) and (2b) are valid for unstrained/unstressed state for $t \leq 0$. If $e(0)=e_{0} \neq 0$ the term $R(t) e_{0}$ has to be added in Eq. (2a) or if $s(0)=s_{0} \neq 0$ the term $C(t) s_{0}$ has to be added in Eq. (2b). In the following, without any loss of generality, we suppose that $e_{0}=0$ and $s_{0}=0$.

Substitution of Eq. (1) in Eqs. (2a) and (2b) leads to constitutive laws that involve fractional operators, namely derivatives and integrals of real order ([7], [8]). This is straightforward for the case in which a strain history is applied (Eq. (2a)) and we want to evaluate the corresponding stress history:

$s(t)=\frac{C_{\rho}}{\Gamma(1-\rho)} \int_{0}^{t}(t-\tau)^{-\rho} \dot{e}(\tau) d \tau=C_{\rho}\left({ }_{0}^{C} D_{t}^{\rho} e\right)(t)$

In Eq. (3) the symbol $\left({ }_{0}^{C} D_{t}^{\rho}\right.$. $)$ represents the Caputo fractional derivative ([7]) of order $\rho$, that is a convolution integral with a power law kernel. In the following sections we will refer to it as $\left(D^{\rho}\right.$.). If we consider the case in which a stress history is applied (Eq. (2b)), integrating by parts and after some manipulations we obtain the Riemann-Liouville (RL) fractional integral of order $\rho\left({ }_{0} D_{t}^{-\rho} \cdot\right)([7])$ :

$$
\begin{aligned}
e(t) & =\frac{1}{C_{\rho} \Gamma(1+\rho)} \int_{0}^{t}(t-\tau)^{\rho} \dot{s}(\tau) d \tau=\frac{1}{C_{\rho} \Gamma(\rho)} \int_{0}^{t}(t-\tau)^{\rho-1} s(\tau) d \tau \\
& =\frac{1}{C_{\rho}}\left({ }_{0} D_{t}^{-\rho} s\right)(t)
\end{aligned}
$$

In the following we will refer to the RL fractional integral as $D^{-\rho}$. The constitutive laws in Eq. (3) and Eq. (4) represent the response of a "springpot" element ([37]). It has been shown in Ref. [9] that the behaviour of the springpot can be reproduced in a classical viscoelasticity framework by an infinite sequence of massless laminae linked by springs/dashpots and laying in a bed of dashpots/springs. This is the reason why the use of fractional viscoelasticity results in a significant reduction of mechanical parameters compared to using calssical viscoelastic models.In order to model the isotropic three-dimensional behaviour of the springpot, it is sufficient to define two relaxation (or creep) functions. The most convenient choice is to use volumetric and deviatoric relaxation (or creep) functions. The relaxation matrix can be written as follows:

$R_{i j k h}(t)=\left(K_{R}(t)-\frac{2}{3} G_{R}(t)\right) \delta_{i j} \delta_{k h}+G_{R}(t)\left(\delta_{i k} \delta_{j h}+\delta_{i h} \delta_{j k}\right)$

where $\delta_{i j}$ is the Kronecker symbol. For both deviatoric $G_{R}(t)$ and volumetric relaxation functions $K_{R}(t)$, power law functions analogous to first of Eq. (1) are selected:

$G_{R}(t)=\frac{G_{\alpha} t^{-\alpha}}{\Gamma(1-\alpha)}$

$K_{R}(t)=\frac{K_{\beta} t^{-\beta}}{\Gamma(1-\beta)}$

where $K_{\alpha}$ and $G_{\beta}$ are anomalous bulk and shear relaxation moduli, respectively, while $\alpha$ and $\beta$ are real numbers indicating the orders of bulk and shear power laws, respectively. 
Table 1

Fractional viscoelastic models.

\begin{tabular}{|c|c|c|c|c|}
\hline Model & Sketch & Constitutive law & $\mathrm{R}(t)$ & $\mathrm{C}(t)$ \\
\hline Springpot & & $s=C_{\rho}\left(D^{\rho} e\right)$ & $\frac{C_{\rho} t^{-\rho}}{\Gamma(1-\rho)}$ & $\frac{t^{\rho}}{C_{\rho} \Gamma(1+\rho)}$ \\
\hline FKV & & $s=E e+C_{\rho}\left(D^{\rho} e\right)$ & $E+\frac{C_{\rho} t^{-\rho}}{\Gamma(1-\rho)}$ & $\frac{1}{E}-\frac{1}{E} E_{\rho}\left(-\frac{E}{C_{\rho}} t^{\rho}\right)$ \\
\hline FM & & $\left(D^{\rho} s\right)+\frac{E}{C_{\beta}} s=E\left(D^{\rho} e\right)$ & $E E_{\rho}\left(-\frac{E}{C_{\rho}} t^{\rho}\right)$ & $\frac{1}{E}+\frac{t^{\rho}}{C_{\rho} \Gamma(1+\rho)}$ \\
\hline FSLS 1 & & $s+\frac{C_{\rho}}{E_{1}+E_{2}}\left(D^{\rho} s\right)=\frac{E_{1} E_{2}}{E_{1}+E_{2}} e+\frac{C_{\rho} E_{1}}{E_{1}+E_{2}}\left(D^{\rho} e\right)$ & $\frac{E_{1} E_{2}}{E_{1}+E_{2}}+\frac{E_{1}^{2}}{E_{1}+E_{2}} E_{\rho}\left(-\frac{\left(E_{1}+E_{2}\right) t^{\rho}}{C_{\rho}}\right)$ & $\frac{E_{1}+E_{2}}{E_{1} E_{2}}-\frac{1}{E_{2}} E_{\rho}\left(-\frac{E_{2}}{C_{\rho}} t^{\rho}\right)$ \\
\hline FSLS 2 & & $s+\frac{C_{\rho}}{E_{2}}\left(D^{\rho} s\right)=E_{1} e+\frac{\left(E_{1}+E_{2}\right) C_{\rho}}{E_{2}}\left(D^{\rho} e\right)$ & $E_{1}+E_{2} E_{\rho}\left(-\frac{E_{2}}{C_{\rho}} t^{\rho}\right)$ & $\frac{1}{E_{1}}-\frac{E_{2}}{E_{1}\left(E_{1}+E_{2}\right)} E_{\rho}\left[-\frac{E_{1} E_{2} t^{\rho}}{\left(E_{1}+E_{2}\right) C_{\rho}}\right]$ \\
\hline
\end{tabular}

By assuming deviatoric and volumetric relaxation functions with the form of Eqs. (6a) and (6b), a four parameter mechanical model is obtained. The strain-stress relationship can be obtained simply by substituting Eq. (5) into the following:

$\boldsymbol{\sigma}(t)=\int_{0}^{t} \boldsymbol{R}(t-\tau) \dot{\varepsilon}(\tau) d \tau$

where $\quad \sigma^{T}(t)=\left[\sigma_{11} \sigma_{22} \sigma_{33} \tau_{12} \tau_{13} \tau_{23}\right] \quad$ and $\quad \boldsymbol{\varepsilon}^{T}(t)=$ $\left[\varepsilon_{11} \varepsilon_{22} \varepsilon_{33}, \gamma_{12} \gamma_{13} \gamma_{23}\right]$ are the stress and strain vectors, respectively, and $\boldsymbol{R}(t)$ is the relaxation matrix in Eq. (5). Since $\boldsymbol{R}(t)$ contains power law functions, the components of the stress vector $\sigma(t)$ depend on the fractional derivatives of the components of the strain vector $\varepsilon(t)$ :

$\sigma_{i i}(t)=2 G_{\alpha}\left(D^{\alpha} \widetilde{\varepsilon}_{i i}\right)(t)+3 K_{\beta}\left(D^{\beta} \bar{\varepsilon}\right)(t) \quad i=1,2,3 ;$

$\tau_{i j}(t)=G_{\alpha}\left(D^{\alpha} \gamma_{i j}\right)(t) ; \quad i, j=1,2,3 ; \quad i \neq j$

where $\bar{\varepsilon}(t)$ is the mean value of the strain $\left(\bar{\varepsilon}=\left(\sum_{i=1}^{3} \varepsilon_{i i}\right) / 3\right)$ and $\widetilde{\varepsilon}_{i i}(t)=\varepsilon_{i i}(t)-\bar{\varepsilon}(t)$. The inverse relationships of Eqs. (8a) and (8b) are readily obtained as follows:

$\varepsilon_{i i}(t)=\frac{1}{2 G_{\alpha}}\left(D^{-\alpha} \tilde{\sigma}_{i i}\right)(t)+\frac{1}{3 K_{\beta}}\left(D^{-\beta} \bar{\sigma}\right)(t) \quad i=1,2,3 ;$

$\gamma_{i j}(t)=\frac{1}{G_{\alpha}}\left(D^{-\alpha} \tau_{i j}\right)(t) ; \quad i, j=1,2,3 ; \quad i \neq j$

where $\bar{\sigma}$ is the mean value of the stress (volumetric component $\bar{\sigma}=$ $\left(\sum_{i=1}^{3} \sigma_{i i}\right) / 3$ and $\tilde{\sigma}(t)$ is the deviatoric component $\tilde{\sigma}(t)=\sigma_{i i}(t)-\bar{\sigma}(t)$.

Eqs. (8a), (8b), (9a) and (9b) have been obtained assuming that the volumetic and the deviatoric creep/relaxation functions are both well fitted by pure power law function and then their behaviour can be reproduced by the springpot model. However, in many cases of engineering interest, the springpot can result not adequate to reproduce completely the time dependent features of viscoelastic materials, then other fractional viscoelastic material models may be used; such an example, the shear creep of some soils is well fitted by the FKV model [6]. Furthermore, several types of polyethylene such as UHMWPE are modelled with the FM model $[19,41,42]$ and, a range of biological tissues are modeled by means of the FSLS model [28]. Moreover it is not excluded that for some materials the volumetric behaviour is well fitted by one fractional viscoelastic model while the deviatoric one is reproduced by using a different fractional viscoelastic model. For these reasons, in the next sections we will introduce other fractional models mentioned above. However, for the sake of clarity, we will first introduce the numerical implementation of the springpot model.

\section{Numerical implementation of the 3D springpot}

Viscoelastic constitutive laws are time dependent equations that in the frame of finite element method (FEM) are solved step by step through numerical integration schemes. Most of the finite element codes allow for the solution of time-dependent problem with two alternative schemes: implicit (Newton-Raphson algorithm) and explicit. We show here details of the implementation of the 3D springpot model in a user material routine into the commercial FE software ABAQUS both in an implicit (UMAT) and explicit scheme (VUMAT). For the implementation of fractional viscoelasticity in an implicit integration scheme, all of the components of stress and the Jacobian at the end of a time step must be provided for each Gauss point. For the implementation in an explicit integration scheme only the components of stress must be provided.

In order to implement these equation in a FE code, the fractional derivatives must be discretized; to this purpose the Grunwald-Letnikov (GL) [7] fractional derivative may be used:

$\left({ }_{0}^{G L} D_{t}^{\rho} f\right)(t)=\left({ }_{0}^{G L} D_{t}^{\rho} f\right)(k \Delta t)=\lim _{\Delta t \rightarrow 0} \Delta t^{-\rho} \sum_{j=1}^{k+1} \lambda_{j}^{(\rho)} f^{(k-j+2)}$

$\lambda_{j+1}^{(\rho)}=\frac{j-1-\rho}{j} \lambda_{j} ; \quad \lambda_{1}=1$

where $f^{(k-j+2)}=f[(k-j+1) \Delta t]$. For sufficiently small $\Delta t$ the GL fractional derivative coincides with the Caputo's fractional derivative; in practical applications, it is not possible to know the sufficiently small time step value a priori. However, it can be determined by analyzing the convergence of the result for decreasing magnitude of $\Delta t$; indeed 
Table 2

\begin{tabular}{lll} 
Implementation of fractional viscoelastic models. \\
\hline Model & Element & Expression \\
\hline springpot & $A_{\rho}^{(k+1)}[e]$ & $C_{\rho} \Delta t^{-\rho} \sum_{j=1}^{k+1} \lambda_{j}^{(\rho)} e^{(k-j+2)}$ \\
& $J_{\rho}$ & $C_{\rho} \Delta t^{-\rho}$ \\
FKV & $A_{\rho}^{(k+1)}[e]$ & $E e^{(k+1)}+C_{\rho} \Delta t^{-\rho} \sum_{j=1}^{k+1} \lambda_{j}^{(\rho)} e^{(k-j+2)}$ \\
& $J_{\rho}$ & $E+C_{\rho} \Delta t^{-\rho}$ \\
FM & $A_{\rho}^{(k+1)}[e, s]$ & $J_{\rho}\left(e^{(k+1)}-\frac{\Delta t^{\rho}}{C_{\rho}} \sum_{j=2}^{k+1} \lambda_{j}^{-(\rho)} s^{(k-j+2)}\right)$ \\
& $J_{\rho}$ & $\frac{E C_{\rho}}{E \Delta t^{\rho}+C_{\rho}}$ \\
& $A_{\rho}^{(k+1)}[e, s]$ & $J_{\rho} e^{(k+1)}+\frac{C_{\rho} \Delta t^{-\rho}}{E_{1}+E_{2}+C_{\rho} \Delta t^{-\rho}} \sum_{j=2}^{k+1} \lambda_{j}^{(\rho)}\left(E_{1} e^{(k-j+2)}-s^{(k-j+2)}\right)$ \\
FSLS 1 & $\frac{E_{1}\left(E_{2}+C_{\rho} \Delta t^{-\rho}\right)}{E_{1}+E_{2}+C_{\rho} \Delta t^{-\rho}}$ \\
& $J_{\rho}$ & $J_{\rho} e^{(k+1)}+\frac{C_{\rho} \Delta t^{-\rho}}{E_{2}+C_{\rho} \Delta t^{-\rho}} \sum_{j=2}^{k+1} \lambda_{j}^{(\rho)}\left[\left(E_{1}+E_{2}\right) e^{(k-j+2)}-s^{(k-j+2)}\right]$ \\
& $A_{\rho}^{(k+1)}[e, s]$ & $\frac{E_{1} E_{2}+\left(E_{1}+E_{2}\right) C_{\rho} \Delta t^{-\rho}}{E_{2}+C_{\rho} \Delta t^{-\rho}}$ \\
\hline
\end{tabular}

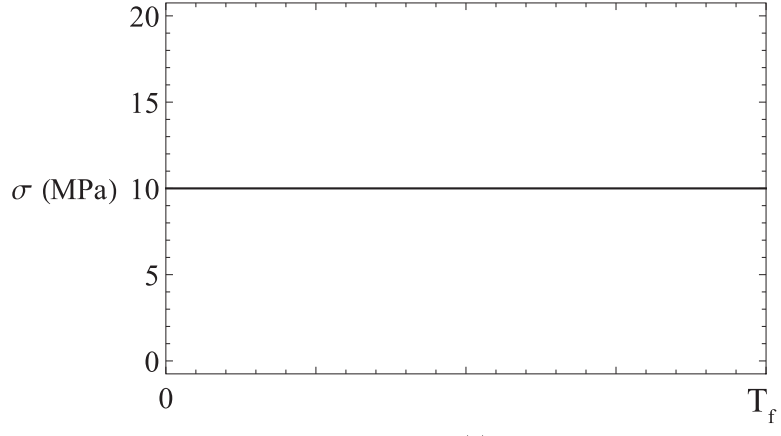

$\mathrm{t}(\mathrm{s})$

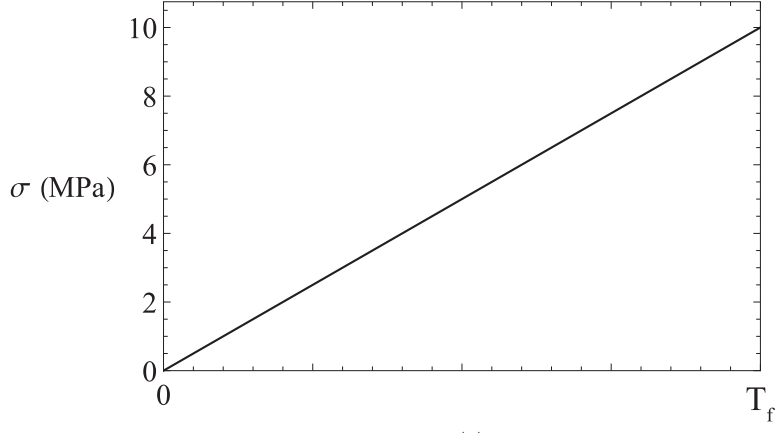

$\mathrm{t}(\mathrm{s})$

(a)

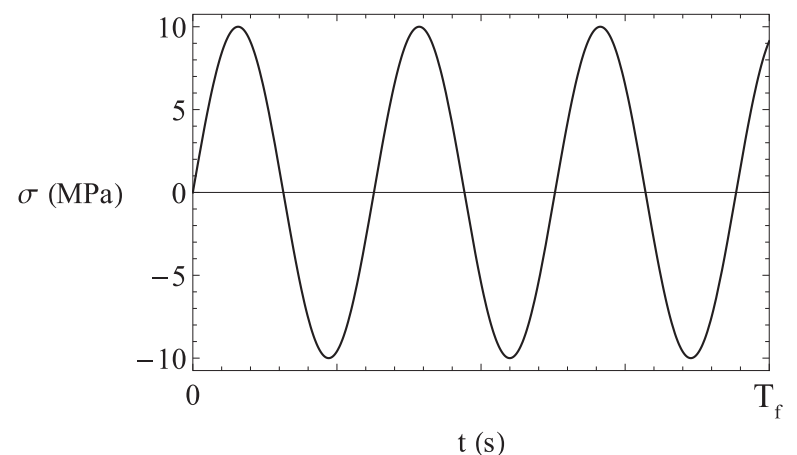

(c)

Fig. 1. Applied stress hisotries of Eqs. (20a), (20b), (20c): constant (a), linear ramp (b) and sinusoidal (c).

there is not a general rule but it depends of the function at hand. For negative values of $\rho$, and small $\Delta t$, the GL operator reverts to the RL fractional integral.

When the FE software calls the UMAT (or VUMAT) the strain at the end of the time increment is already known and the corresponding stress has to be evaluated. If the constitutive model of Eqs. (8a) and (8b) is assumed, by using the GL fractional derivative the stress at the end of the $k$ - th time increment $(t=k \Delta t)$ may be written as:

$\sigma_{i i}^{(k+1)}=2 A_{\alpha}^{(k+1)}\left[\widetilde{\varepsilon}_{i i}\right]+3 A_{\beta}^{(k+1)}[\bar{\varepsilon}] ; \quad i=1,2,3 ;$

$\tau_{i j}^{(k+1)}=A_{\alpha}^{(k+1)}\left[\gamma_{i j}\right] ; \quad i, j=1,2,3 ; \quad i \neq j$

where $A_{\rho}^{(k+1)}$ are operators dependent on the fractional order $\alpha$ and $\beta$ and on the other mechanical parameters. For the three-dimensional springpot model (Eqs. (8a), (8b), (9a) and (9b)) these operators are given as:

$A_{\rho}^{(k+1)}[e]=C_{\rho} \Delta t^{-\rho} \sum_{j=1}^{k+1} \lambda_{j}^{(\rho)} e^{(k-j+2)} \quad i=1,2,3 ;$

Such an example, the term $A_{\alpha}^{(k+1)}\left[\widetilde{\varepsilon}_{i i}\right]$ in Eq. (11a) is returned by Eq. (12) if $e, \rho$ and $C_{\rho}$ are replaced by $\widetilde{\varepsilon}_{i i}, \alpha$ and $G_{\alpha}$, respectively. The components of the Jacobian may be easily evaluated as:

$\frac{\partial \Delta \sigma_{i j}^{(k+1)}}{\partial \Delta \varepsilon_{k h}^{(k+1)}}=\left(J_{\beta}-\frac{2}{3} J_{\alpha}\right) \delta_{i j} \delta_{k h}+J_{\alpha}\left(\delta_{i k} \delta_{j h}+\delta_{i h} \delta_{j k}\right)$ 


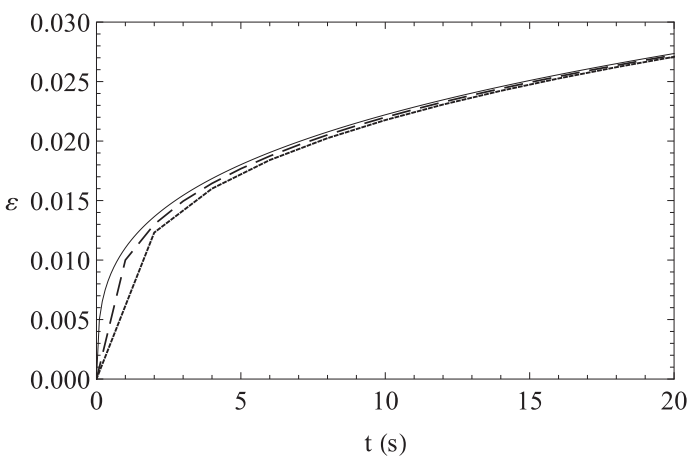

(a)

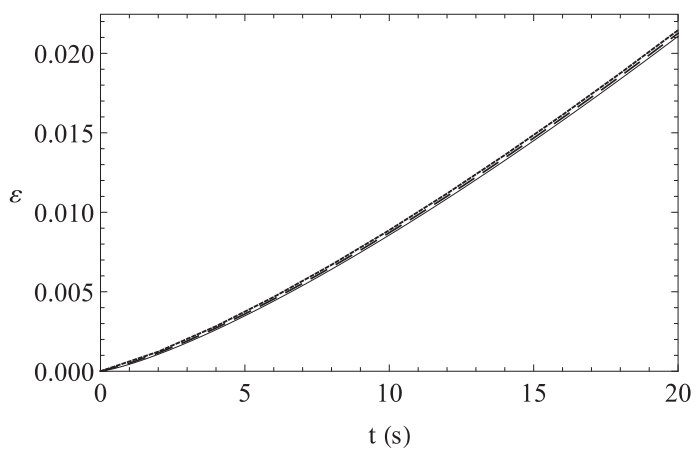

(b)

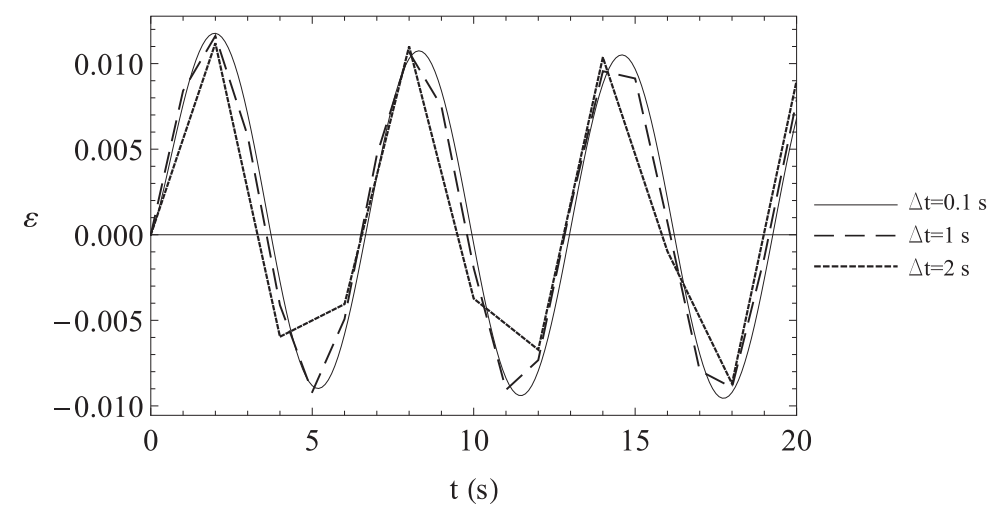

(c)

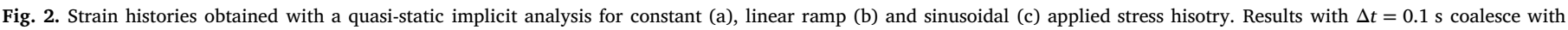
analytical results.

For the sake of clarity:

$$
\begin{aligned}
& \frac{\partial \Delta \sigma_{i i}^{(k+1)}}{\partial \Delta \varepsilon_{i i}^{(k+1)}}=J_{\beta}+\frac{4}{3} J_{\alpha} ; \quad i=1,2,3 ; \\
& \frac{\partial \Delta \sigma_{i i}^{(k+1)}}{\partial \Delta \varepsilon_{j j}^{(k+1)}}=J_{\beta}-\frac{2}{3} J_{\alpha} \quad i, j=1,2,3 ; \quad i \neq j \\
& \frac{\partial \Delta \tau_{i j}^{(k+1)}}{\partial \Delta \gamma_{i j}^{(k+1)}}=J_{\alpha} \quad i, j=1,2,3 ; \quad i \neq j
\end{aligned}
$$

where

$J_{\rho}=C_{\rho} \Delta t^{-\rho}$

As an example, the term $J_{\beta}$ is returned by Eq. (15) if $C_{\rho}$ and $\rho$ are replaced by $K_{\beta}$ and $\beta$, respectively. It is to be noted that the Jacobian depends only on the value of $\Delta t$ and on the mechanical parameters.

Some commercial FE codes that use an implicit Newton-Raphson integration scheme allow the time increment to be determined automatically to optimize the run time. The GL formula for evaluating of the fractional derivatives has been derived assuming a constant increment (i.e. the time) and, to the best of our knowledge, a corresponding formulation for a variable increment is not available in the literature; furthermore, the automatic time increment requires the definition of a tolerance criterion, that is difficult to define without knowledge of the elastic and inelastic parts of the strain. For these two reasons we have currently limited ourselves to using this model with a fixed time increment. In order to evaluate the GL derivative the history of strain at each Gauss Point must be stored leading possibly to a considerable amount of memory when analysing large FE models. Hence a number of strategies to overcome this problem have been explored and are discussed in Section 5 .
In the next section the fractional Kelvin Voigt (FKV), the fractional Maxwell (FM) and the fractional standard linear solid (FSLS) models are introduced and their implementation in user material routines is presented.

\section{Other fractional constitutive models}

So far we have presented the details of the formulation of the simple springpot model in three-dimensional fractional constitutive model. In particular, it has been assumed that volumetric and deviatoric components of the stress are power laws with different time scales:

$\bar{\sigma}(t)=3 K_{\beta}\left(D^{\beta} \bar{\varepsilon}\right)(t) ; \quad \tau_{i j}=G_{\alpha}\left(D^{\alpha} \gamma_{i j}\right)(t)$

However, in some cases of practical interest, it is necessary to introduce more complicated fractional viscoelastic models which may give a better representation of real viscoelastic materials. For example, the volumetric component of the creep function expressed with a single springpot gives rise to a non-physical situation. Namely, under a constant hydrostatic pressure at $t=\infty$, the material will collapse to a single point. In order to overcome to this undesired feature some authors add one or more elastic terms into the fractional differential equation. It is not clear the correct way to define the elastic part in the constitutive equations of a fractional viscoelastic model, but it is known that the viscoelastic behaviour of some material is well reproduced by means of models like the FKV, the FM and the FSLS models (see Refs. $[6,17,19,28,32])$.

In Table 1 the constitutive laws for the generic stress $s(t)$ (volumetric or deviatoric) and the corresponding strain $e(t)$ (volumetric or deviatoric) as well as the creep and relaxation functions are reported for the most common models: the FKV, the FM and two FSLS models. 


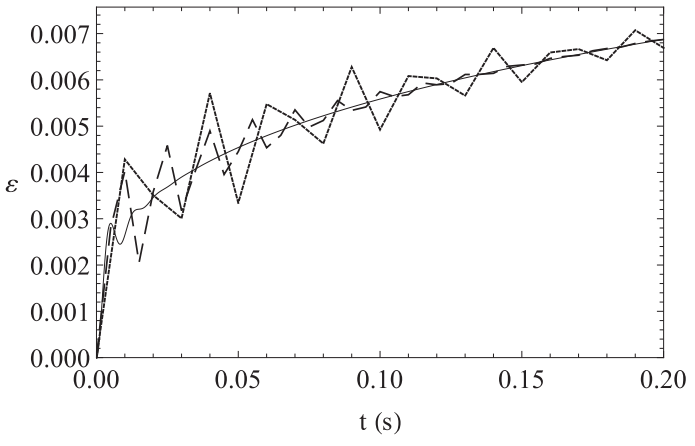

(a)

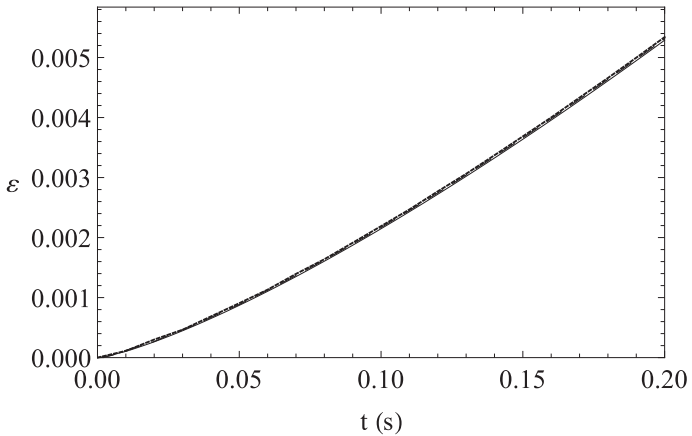

(b)

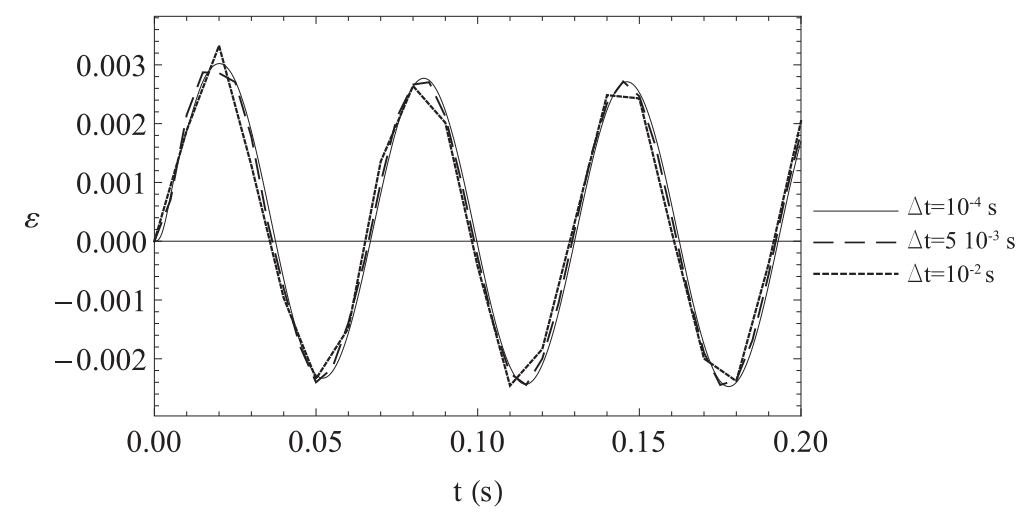

(c)

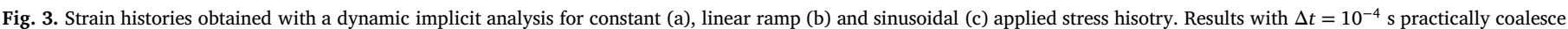
with analytical results.

The two FSLS models (FSLS1 and FSLS2) are equivalent, but since they are both used in literature, their implementation has been included.In Table $1 E_{\rho}(\cdot)$ is the one parameter Mittag-Leffler function defined as follows

$E_{\rho}(z)=\sum_{j=0}^{\infty} \frac{z^{j}}{\Gamma(\rho j+1)}$

Any of the three dimensional fractional viscoelastic models can be reconstructed by properly selecting the constitutive laws in Table 1. Let us assume, for example, that the volumetric component is a fractional $\mathrm{KV}$ element characterized by $K_{\beta}, \beta$ and $K$ and the deviatoric one is a pure springpot characterized by $G_{\alpha}$ and $\alpha$. Then the three dimensional fractional constitutive laws are given as:

$\sigma_{i i}(t)=2 G_{\alpha}\left(D^{\alpha} \widetilde{\varepsilon}_{i i}\right)(t)+3 K \bar{\varepsilon}(t)+3 K_{\beta}\left(D^{\beta} \bar{\varepsilon}\right)(t) ; \quad i=1,2,3 ;$

$\tau_{i j}(t)=G_{\alpha}\left(D^{\alpha} \gamma_{i j}\right)(t) ; \quad i, j=1,2,3 ; \quad i \neq j$

The implementation of all of the models of Table 1 can be performed in a way analogous to the procedure of Section 3; indeed, by discretizing the fractional derivatives in the constitutive equations of Table 1 the stress at the end of the time increment of the FE simulation can be found in terms of the history of strain. When the FM is selected, the history of stress instead of the history of strain is needed; when one of the FSLS models is chosen, the stress at the end of the time increment depends on both the history of stress and the history of strain; for this reason Eqs. (11a) and (11b) are slightly modified as follows:

$\sigma_{i i}^{(k+1)}=2 A_{\alpha}^{(k+1)}\left[\widetilde{\varepsilon}_{i i}, \frac{\tilde{\sigma}_{i i}}{2}\right]+3 A_{\beta}^{(k+1)}\left[\bar{\varepsilon}, \frac{\bar{\sigma}}{3}\right] ; \quad i=1,2,3 ;$

$\tau_{i j}^{(k+1)}=A_{\alpha}^{(k+1)}\left[\gamma_{i j}, \tau_{i j}\right] ; \quad i, j=1,2,3 ; \quad i \neq j$
This fact implies that for the FSLS models the amount of memory required to evaluate the increment of stress is double than the memory required for the springpot, the FKV and FM models.

Moreover, since the fractional models have different constitutive equations, the operator $A_{\rho}^{(k+1)}$ and the expressions for the components of the Jacobian are different from those described in Eqs. (11a), (11b), (14a), (14b) and (14c). In Table 2 the expression for $A_{\rho}^{(k+1)}$ and $J_{\rho}$ are summarized for all the fractional models treated in this work. Note that only in the springpot and in the FKV model the operator $A_{\rho}^{(k+1)}$ does not depend on the stress history. For the implementation of fractional three dimensional models with different definition of the volumetric and deviatoric contribution (see for example [32]), Eqs. (11a) and (11b) (or Eqs. (19a) and(19b)) and Eqs. (14a), (14b) and (14c) remain valid, while the definitions of the operators $A_{\rho}^{(k+1)}$ and the components of the Jacobian $J_{\rho}$ change according to Table 2. The relationships of Table 2 demonstrate how the use of GL fractional derivatives allows the unknown stresses to be determined also when a simple analytical solution in terms of stress is not available. Then the implementation of convolution integrals with Mittag-Leffler function kernels as done in Ref. [28] is avoided. Furthermore, the evaluation of the components of the Jacobian is straightforward and leads to simple expressions which are indeed suitable for implementation purposes and since they depend only on the time increment and the mechanical parameters, they can be evaluated only once during the FE simulation. As it can been from Table 2 the implementation of fractional viscoelastic constitutive models is straightforward also for researchers and engineers not expert on fractional calculus.

The routines for the numerical implementation in FE framework are efficient since they involve only summation of products; the time needed for the evaluation of the solution through a single time interval is different for each time interval and in particular the most demanding time step is the last, because the number of terms in the summation of 
the operator $A_{\rho}^{(k+1)}$ is $k$. This means that for fixed time increment and total time of simulation, the time needed for the analysis is approximatively proportional to the square of the number of time increment in which the time of observation is discretized.

The most important issue in the implementation of fractional viscoelastic laws is that we need to have access to and store the history of strains (and/or stresses) in order to obtain the increment of stress (and/or strain). This could potentially lead to a considerable amount of memory required to run large simulations. In the next section we discuss some strategies in order to reduce the memory required to run simulations with large number of FEs.

\section{Hereditariness and related computational problems}

The implementation of fractional viscoelastic models require storing the history of strain at each gauss point. In order to satisfy this requirement, we store the values of the components of strain at each increment in a Common Block (CB) (available in FORTRAN programming language). The $\mathrm{CB}$ is a piece of shared memory that allows us to pass information between program units. Another strategy may be to store the past history of strain/stress in a text file. However, in this way the routines are much slower and for this reason is preferable to use the $\mathrm{CB}$. In order to reduce the amount of memory two possible strategies are proposed:

- use a larger time increment $\Delta t$;

- "truncate" the memory of the material.

The first option discussed in Section 5.1 is only applicable to implicit analysis, because in explicit analysis the solution diverges when the time increment is not sufficiently small. The second strategy is applicable only to explicit analysis and will be discussed in Section 5.2.

Another possible way to limit the memory needed for the simulation is to use FEs of lower integration order which have a reduced number of Gauss points. In the case of fractional viscoelasticity using more FEs could lead to a fewer integration points in the structural model and, as a consequence, to a reduced memory needed during the simulation.

\subsection{Reducing the amount of memory required in implicit analysis (UMAT)}

Implicit analysis uses the Newton-Raphson iterative algorithm to find the solution with a prescribed accuracy. This allows the analysis to run with a time increment larger than the time increment needed to perform correctly the integration of a fractional differential equation (which leads to the exact solution). In other words, in the simple case of Fig. 3 a $\Delta t=T_{f} / 1000 ; s$ is needed to obtain the analytic solution (i.e.perform correctly the integration of a fractional differential equation). However a $\Delta t=T_{f} / 10 ; s$ would still be adequate. Choosing a larger $\Delta t$ obviously costs in terms of accuracy. In the case of explicit analysis a time increment of $\Delta t=T_{f} / 100 ; s$ is the largest value that can be used in order to obtain a solution which converges to the exact solution. The amount of memory used by the analysis can be significantly reduced depending on the accuracy required. In order to show this, some tests have been performed with a one FE model of a truss forced by different stress histories; results remain valid also for bi- and three-dimensional models. The material is a springpot with $\rho=0.3$ and $C_{\rho}=10^{3} \mathrm{MPas}^{\rho}$. The applied histories of stress are the following:

$\sigma(t)=\sigma_{0} U(t)$

$\sigma(t)=\sigma_{0} \frac{t}{T_{f}}$

$\sigma(t)=\sigma_{0} \sin (\omega t)$

where $\sigma_{0}=1 \mathrm{MPa}, T_{f}$ is the final time of the analysis and $\omega$ is the circular frequency of the sinusoidal load. The stress histories of Eqs.
Table 3

Mean percentage errors on predicted strains for the three stress histories considered in the implicit quasi-static analysis.

\begin{tabular}{llll}
\hline$\Delta t(s)$ & $\sigma_{0} U(t)$ & $\sigma_{0} \mathrm{t} / \mathrm{T}_{f}$ & $\sigma_{0} \sin (\omega t)$ \\
\hline 0,1 & 0,28 & 0,49 & 1,06 \\
1 & 1,86 & 3,23 & 5,48 \\
2 & 3,04 & 5,22 & 103,73 \\
\hline
\end{tabular}

Table 4

Percentage errors on predicted strain at the end of the analysis for the three stress histories considered in the implicit quasi-static analysis.

\begin{tabular}{llll}
\hline$\Delta t(s)$ & $\sigma_{0} U(t)$ & $\sigma_{0} \mathrm{t} / \mathrm{T}_{f}$ & $\sigma_{0} \sin (\omega t)$ \\
\hline 0,1 & 0,05 & 0,09 & 0,13 \\
1 & 0,52 & 0,96 & 5,03 \\
2 & 1,04 & 1,91 & 32,77 \\
\hline
\end{tabular}

(20a), (20b) and (20c) are depicted in Fig. 1. For a quasi-static analysis, where inertial forces are neglected, $T_{f}=20 \mathrm{~s}$ and $\omega=1 \mathrm{rad} / \mathrm{s}$ are chosen; the analysis has been performed with three different time increments and results are compared in terms of obtained strain histories. From Fig. 2 it is evident that, for all three applied stress histories in Fig. 1, the results at the end of the analysis may be considered good also for the larger time increment used that is $\Delta t=2 \mathrm{~s}$. Indeed, the choice of a larger time increment allows to save memory. However, it is worth noting that in the case of a sinusoidal applied history Fig. 2 c, by choosing larger time increment $\Delta t=2 \mathrm{~s}$ the accuracy of the solution decreases in comparison with the case of Fig. 2 a, b. Tables 3 and 4 show the mean percentage error $E_{m}$ and the percentage error at the last time increment $E_{f}$ of each analysis, respectively; these quantities are defined as follows:

$E_{m}=\frac{1}{N} \sum_{k=1}^{N} \frac{\left|\varepsilon_{k}^{(F E M)}-\varepsilon_{k}^{(a n)}\right|}{\left|\varepsilon_{k}^{(a n)}\right|} \times 100$

$E_{f}=\frac{\left|\varepsilon_{N}^{(F E M)}-\varepsilon_{N}^{(a n)}\right|}{\left|\varepsilon_{N}^{(a n)}\right|} \times 100$

where $\varepsilon_{k}^{(F E M)}$ is the strain at the $k$ - th time increment evaluated with the subroutine, $\varepsilon_{k}^{(a n)}$ is the corresponding analytical strain and $N$ is the number of time increments in which the time of observation is discretized in the FE analysis. The error $E_{f}$ is given because in some situations the user may be interested only to the final state of a FE analysis (or a part of it) and not in the all history of stress/strain. From Tables 3 and 4 it can be concluded that the most demanding situation in implicit quasi-static situation is when a periodic input is applied to the viscoelastic model at hand, while accurate results are achieved more easily when a constant load is applied.

The same tests have been performed in implicit dynamic conditions; in order to observe transitory dynamical effects $T_{f}=0.2 \mathrm{~s}$ and $\omega=100 \mathrm{rad} / \mathrm{s}$ are chosen. Results are reported in Fig. 3. Tables 5 and 6 show the values of $E_{m}$ and $E_{f}$, respectively, for each implicit dynamic analysis. As for the case of quasi-static analysis, a large $\Delta t$ gives always acceptable results, however the strain histories are not reproduced with great accuracy. Differently from quasi-static conditions, in a dynamic implicit analysis, the less demanding situation is when a linear ramp stress is applied. The major difference between quasi-static and dynamic analysis is when a constant stress is applied; indeed, in a dynamical analysis the materials oscillates when the load is applied suddenly, and this oscillations cannot be well reproduced with a large time increment. In the quasi-static analysis, instead, the obtained strain history is well reproduced except at the beginning when results are affected by the fact that the FE software must treat the stress as linear across the first 
Table 5

Mean percentage errors on predicted strains for the three stress histories considered in the implicit dynamic analysis.

\begin{tabular}{llll}
\hline$\Delta t(s)$ & $\sigma_{0} U(t)$ & $\sigma_{0} \mathrm{t} / \mathrm{T}_{f}$ & $\sigma_{0} \sin (\omega t)$ \\
\hline $10^{-4}$ & 0,24 & 0,32 & 4,8 \\
$5 \times 10^{-3}$ & 5,27 & 1,85 & 26,84 \\
$10^{-2}$ & 12,26 & 2,93 & 80,13 \\
\hline
\end{tabular}

Table 6

Percentage errors on predicted strain at the end of the analysis for the three stress histories considered in the implicit dynamic analysis.

\begin{tabular}{llll}
\hline$\Delta t(s)$ & $\sigma_{0} U(t)$ & $\sigma_{0} \mathrm{t} / \mathrm{T}_{f}$ & $\sigma_{0} \sin (\omega t)$ \\
\hline $10^{-4}$ & 0,01 & 0,00 & 1,13 \\
$5 \times 10^{-3}$ & 0,05 & 0,48 & 7,91 \\
$10^{-2}$ & 2,7 & 0,98 & 18,74 \\
\hline
\end{tabular}

time increment, then the load is ramped up linearly over the first time increment.

\subsection{Reducing the amount of memory required in explicit analysis (VUMAT)}

In explicit analysis it is possible to "truncate" the memory of the material. This can be done due to the short memory principle [7] that allows the fractional derivative to be approximated by taking into account a finite memory $M$ :

$\left({ }_{a} D_{t}^{\alpha} f\right)(t) \simeq\left({ }_{M-t} D_{t}^{\alpha} f\right)(t), \quad(t>a+M)$

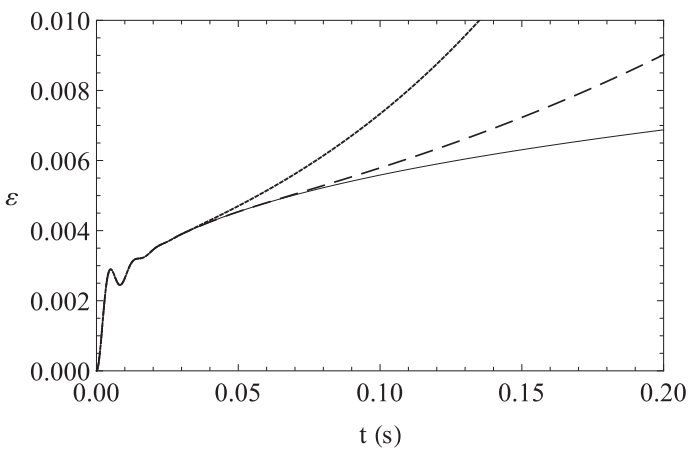

(a)
This imply that for $t>a+M$ the fractional derivative is performed with a moving lower limit and the number of terms of the discretized fractional derivative is not larger that $M / \Delta t$. By this approximation it is possible to limit the amount of memory required for the analysis. This strategy costs, surely, in terms of accuracy; the error committed by "truncating" the memory of the material may be estimated with the strategy described in Ref. [7], where the memory length $M$ is related to the accuracy required, in terms of maximum error allowed. However this is not meaningful for the scope of this work. Instead, the subroutine for the springpot model has been tested with the same model of previous section with $T_{f}=0.2 \mathrm{~s}$ and $\omega=100 \mathrm{rad} / \mathrm{s}$ and with different values of the memory length $M$. Results of Fig. 4 show that in some cases it is possible to truncate the memory of the material without losing the accuracy of the solution. The errors $E_{m}$ and $E_{f}$ are shown in Tables 7 and 8. It is evident that when the applied stress history is a linear ramp, it is possible to save the memory used for the analysis by truncating the memory; even when the memory is $T_{f} / 4$ the results are overall accurate. With an applied constant stress or a sinusoidal history of stress, instead, an acceptable accuracy in terms of obtained strain requires that memory is long at least $T_{f} / 2$. It is to be noted that while in implicit dynamic analysis the most demanding situation is when the applied load is periodic, in explicit dynamic analysis the situation requiring more computational effort is for sure the case with an applied constant stress.

\section{Validation of the code: benchmark problems}

In this section the routines discussed above are tested by running simple problems whose analytical solution is known. In particular in Section 6.1 the FSLS1 is considered while in Section 6.2 the FKV model is used to model the viscoelastic material; the tests with other fractional viscoelastic models are not reported for the sake of brevity. The rou-

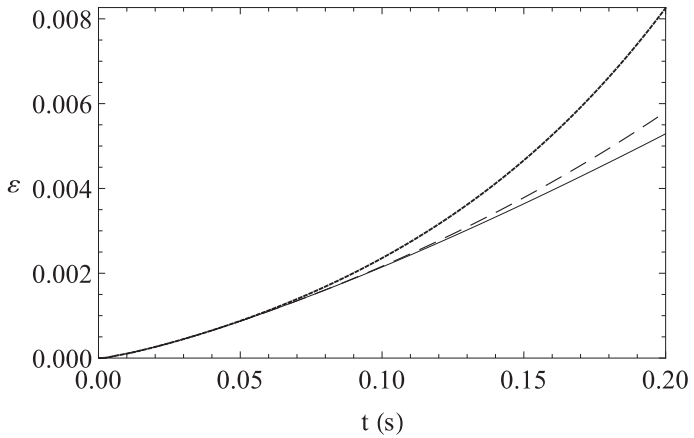

(b)

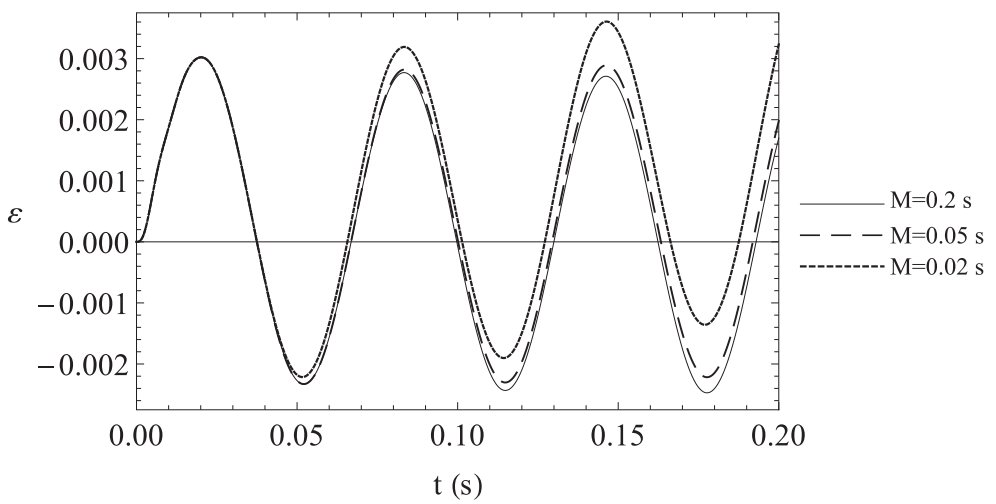

(c)

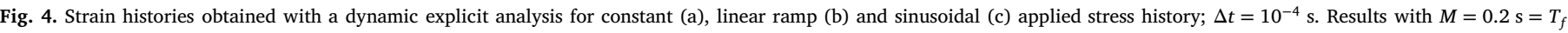
practically coalesce with analytical results. 
Table 7

Mean percentage errors on predicted strains for the three stress histories considered in the explicit dynamic analysis.

\begin{tabular}{llll}
\hline$M(s)$ & $\sigma_{0} U(t)$ & $\sigma_{0} \mathrm{t} / \mathrm{T}_{f}$ & $\sigma_{0} \sin (\omega t)$ \\
\hline 0,2 & 0,24 & 0,31 & 4,77 \\
0,05 & 8,31 & 2,57 & 24,00 \\
0,02 & 47,13 & 16,34 & 117,92 \\
\hline
\end{tabular}

Table 8

Percentage errors on predicted strain at the end of the analysis for the three stress histories considered in the

\begin{tabular}{llll}
\hline$M(s)$ & $\sigma_{0} U(t)$ & $\sigma_{0} \mathrm{t} / \mathrm{T}_{f}$ & $\sigma_{0} \sin (\omega t)$ \\
\hline 0,2 & 0,01 & 0,00 & 1,13 \\
0,05 & 31,19 & 9,80 & 15,08 \\
0,02 & 158,72 & 56,11 & 88,10 \\
\hline
\end{tabular}

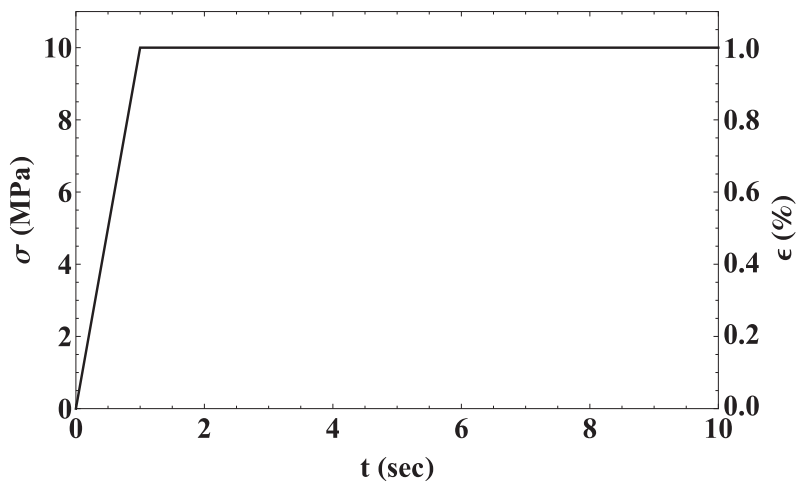

Fig. 6. Stress history (Eq. (23)) and strain history (Eq. (29)) for the creep and relaxation tests, respectively.

tines have been tested with the finite element software ABAQUS 6.14 [38] with both implicit and explicit simulations and they gave the same results in terms of accuracy. However, for the brevity sake, in the following only results of benchmark with implicit quasi-static simulations are reported.

\subsection{Creep with FSLS1 model}

Here we analyze a viscoelastic cube (Fig. 5), made of the FSLS1 model material of Table 1 , subjected to creep tests. The mechanical properties of the cube are: $K_{\beta}=5 \times 10^{8} \mathrm{~Pa} \mathrm{~s}^{\beta}, K_{1}=10^{9} \mathrm{~Pa}$, explicit dynamic analysis.

$K_{2}=5 \times 10^{8} \mathrm{~Pa}, \quad G_{\alpha}=3.75 \times 10^{8} \mathrm{~Pa} \mathrm{~s}^{\alpha}, \quad G_{1}=7.5 \times 10^{8} \mathrm{~Pa}$, $G_{2}=3.75 \times 10^{8} \mathrm{~Pa}$; different values of the parameters $\alpha=\beta$ have been considered: $0,0.25,0.5,0.75,1$. For this model the instantaneous bulk and shear moduli are $K_{1}$ and $G_{1}$, respectively, while the long term bulk and shear moduli are defined as $\frac{K_{1} K_{2}}{K_{1}+K_{2}}$ and $\frac{G_{1} G_{2}}{G_{1}+G_{2}}$, respectively; $K_{\beta}$ and $G_{\alpha}$ are the viscoelastic bulk and shear coefficients, respectively.

The cube has one of its faces normal to the $x$-direction fixed only in the $x$-direction. On the opposite face the stress history of Fig. 6 is applied in the $x$-direction; the final constant value of stress is $\sigma_{x x}=\sigma_{0}=10 \mathrm{MPa}$. This stress history can be written as follow:

$\sigma_{x x}(t)=\sigma_{0}\left[t\left(U(t)-U\left(t-t_{0}\right)\right)+U\left(t-t_{0}\right)\right]$

where $U(\cdot)$ is the Unit step function and $t_{0}=1 \mathrm{~s}$. The analytical solution is easily obtained by using the second of Eq. (2); the creep laws of the FSLS1 model are the following

$K_{C}(t)=\frac{1}{K_{2}}\left[\frac{K_{1}+K_{2}}{K_{1}}-E_{\beta}\left(-\frac{K_{2}}{K_{\beta}} t^{\beta}\right)\right]$

$G_{C}(t)=\frac{1}{G_{2}}\left[\frac{G_{1}+G_{2}}{G_{1}}-E_{\alpha}\left(-\frac{G_{2}}{G_{\alpha}} t^{\alpha}\right)\right]$

By substituting Eqs. (24a), (24b) and (23) in Eqs. (9a) and (9b), the response in terms of strain components is obtained as

$\varepsilon_{x x}(t)=\sigma_{0}\left(L_{1}(t)-L_{1}\left(t-t_{0}\right)\right)$

$\varepsilon_{y y}(t)=\varepsilon_{z z}(t)=\sigma_{0}\left(L_{2}(t)-L_{2}\left(t-t_{0}\right)\right)$

where

$L_{1}(t)=U(t)\left[\frac{\left(I^{1} K_{C}\right)(t)}{9}+\frac{\left(I^{1} G_{C}\right)(t)}{3}\right]$

$L_{2}(t)=U(t)\left[\frac{\left(I^{1} K_{C}\right)(t)}{9}-\frac{\left(I^{1} G_{C}\right)(t)}{6}\right]$

and $\left(I^{1} K_{C}\right)(t)$ and $\left(I^{1} G_{C}\right)(t)$ are the first integrals of the creep functions of Eqs. (24a) and (24b)

$\left(I^{1} K_{C}\right)(t)=t\left[\frac{K_{1}+K_{2}}{K_{1} K_{2}}-\frac{1}{K_{2}} E_{\beta, 2}\left(-\frac{K_{2}}{K_{\beta}} t^{\beta}\right)\right]$

$\left(I^{1} G_{C}\right)(t)=t\left[\frac{G_{1}+G_{2}}{G_{1} G_{2}}-\frac{1}{G_{2}} E_{\alpha, 2}\left(-\frac{G_{2}}{G_{\alpha}} t^{\alpha}\right)\right]$

In $E_{\beta, \phi}(\cdot)$ is the two parameter Mittag-Leffler function defined as

$E_{\beta, \phi}(z)=\sum_{j=0}^{\infty} \frac{z^{j}}{\Gamma(\beta j+\phi)}$

Notice that the one parameter Mittag-Leffler function of Eq. (17) is a particular case of Eq. (28) in which $\phi=1$.
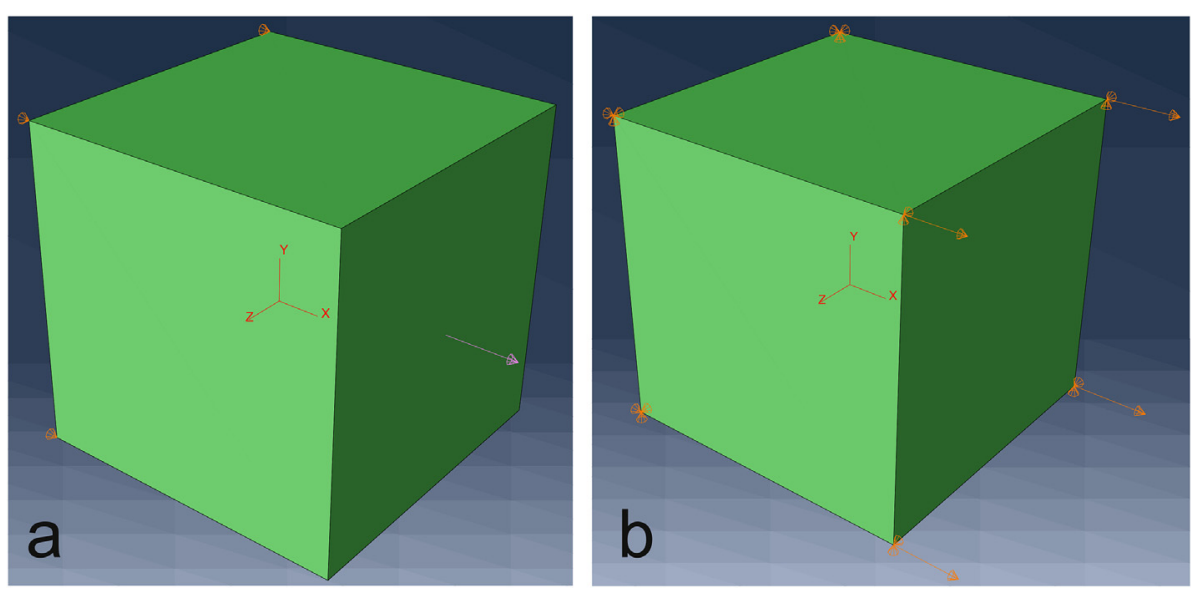

Fig. 5. Viscoelastic cube for the creep test (a) and relaxation test (b). 

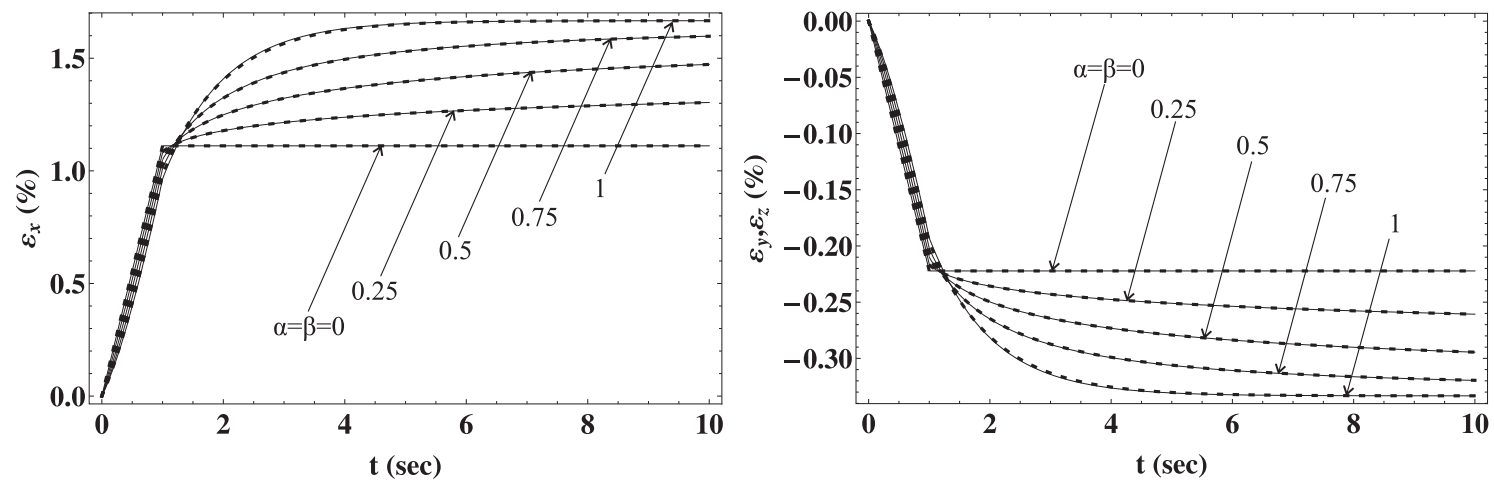

Fig. 7. Comparison between analytical and FEM responses for creep test of the cube in Fig. 5.
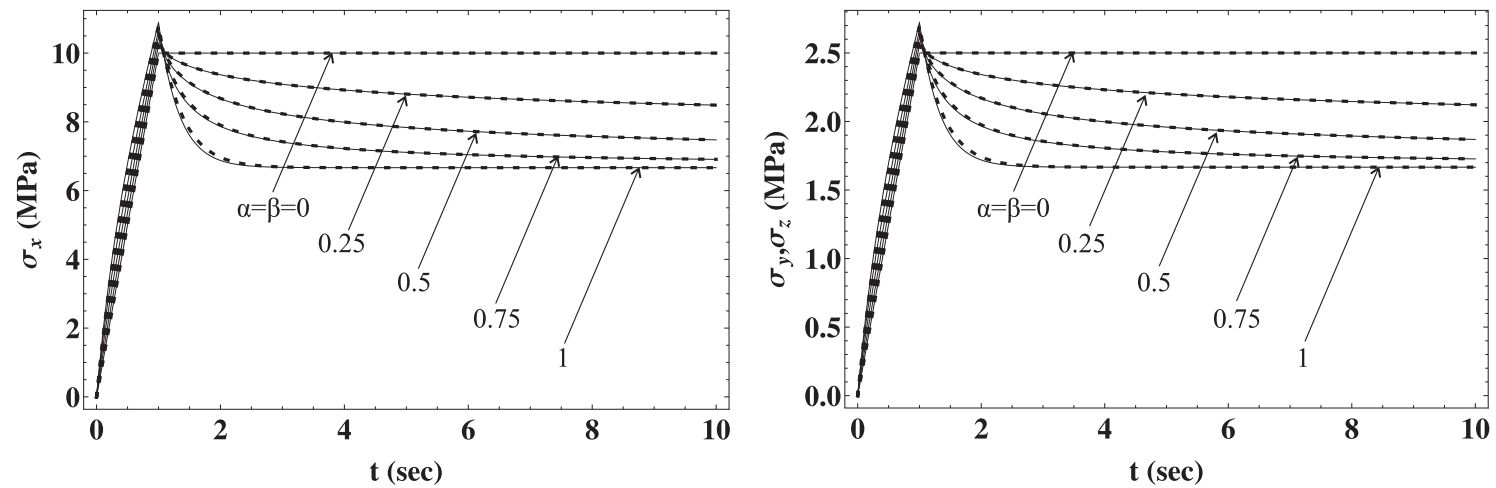

Fig. 8. Comparison between analytical and FEM responses for relaxation test of the cube in Fig. 5b.

Fig. 7 shows the comparison between the FEM result and the analytical solution; red dashed lines are responses evaluated with FEM with a constant time step of $0.1 \mathrm{~s}$, while black continuous lines represent the analytical solutions. From this figure it is possible to appreciate that the solutions are identical.

\subsection{Relaxation test with FSLS1 model}

In the relaxation test in Fig. $5 \mathrm{~b}$ all but one of the faces of the cube are prevented form displacing in the normal direction. The mechanical parameters are the same of the creep test of previous section. A displacement of $1 \mathrm{~mm}$ is applied on the face opposite to the constrained one, that corresponds to a strain $\varepsilon_{x x}=\varepsilon_{0}=0.01=1 \%$ (see Fig. 6); the displacement was applied with a linear ramp of $1 \mathrm{~s}$ and then held for other $9 \mathrm{~s}$ as it is shown in Fig. 6. With the boundary conditions described above $\varepsilon_{y y}(t)=\varepsilon_{z z}(t)=0$ and all the direct components of stress are different from zero. The history of the superimposed strain can be written as follows:

$\varepsilon_{x x}(t)=\varepsilon_{0}\left[t\left(U(t)-U\left(t-t_{0}\right)\right)+U\left(t-t_{0}\right)\right]$

The relaxation function of the FSLS1 model are deducted from Table 1 and are reported here for clarity

$K_{R}(t)=\frac{K_{1}}{K_{1}+K_{2}}\left[K_{1} E_{\beta}\left(-\frac{K_{1}+K_{2}}{K_{\beta}} t^{\beta}\right)+K_{2}\right]$
$G_{R}(t)=\frac{G_{1}}{G_{1}+G_{2}}\left[G_{1} E_{\alpha}\left(-\frac{G_{1}+G_{2}}{G_{\alpha}} t^{\alpha}\right)+G_{2}\right]$

Then by inserting Eqs. (30a), (30b) and (29) in the first of Eqs. (2a) and (2b) the analytical solution is easily obtained as

$\sigma_{x x}(t)=\varepsilon_{0}\left(L_{3}(t)-L_{3}\left(t-t_{0}\right)\right)$

$\alpha=0.5$
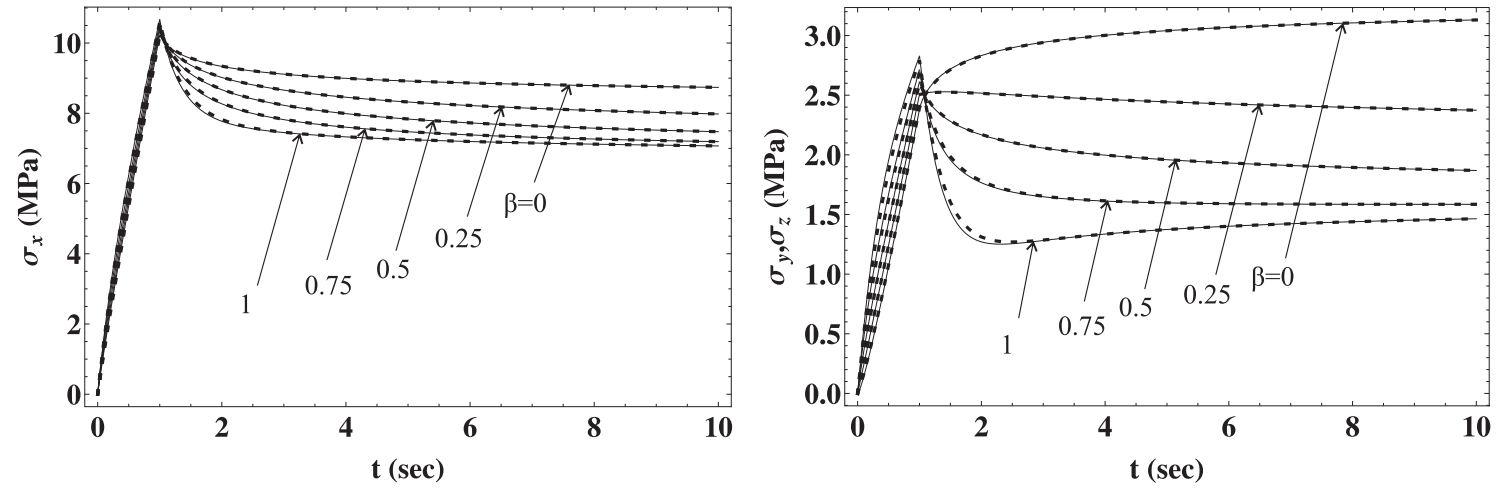

Fig. 9. Comparison between analytical and FEM responses for relaxation test of the cube in Fig. 5b with fixed value of $\alpha$ and different values of $\beta$. 


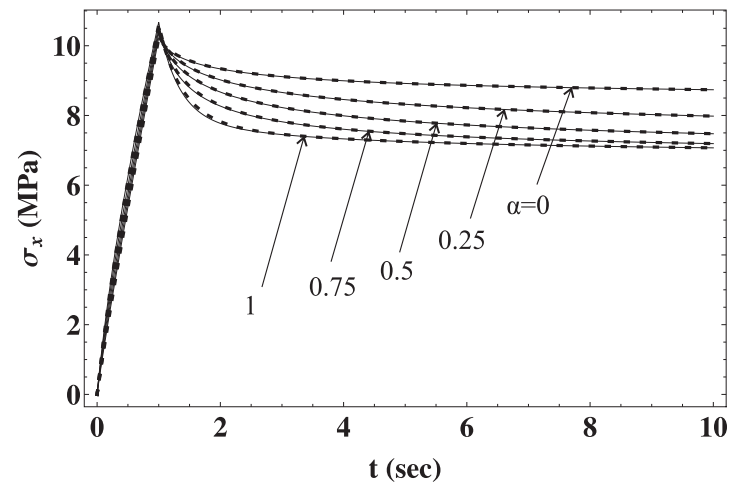

$\beta=0.5$

Fig. 10. Comparison between analytical and FEM responses for relaxation test of the cube in Fig. 5b with fixed value of $\beta$ and different values of $\alpha$.

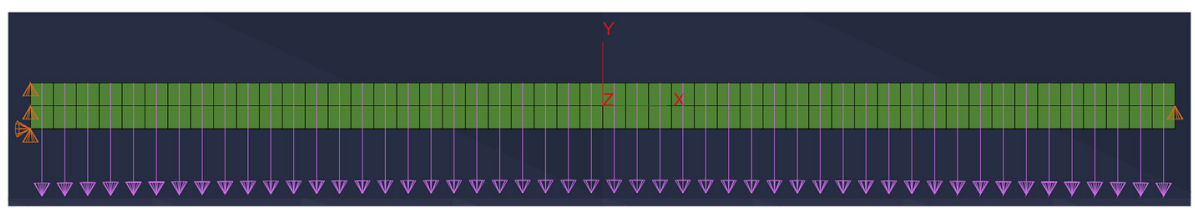

Fig. 11. 2D plain strain model of the Euler-Bernoulli viscoelastic beam.

$\sigma_{y y}(t)=\sigma_{z z}(t)=\varepsilon_{0}\left(L_{4}(t)-L_{4}\left(t-t_{0}\right)\right)$

where

$L_{3}(t)=U(t)\left(\left(I^{1} K_{R}\right)(t)+\frac{4}{3}\left(I^{1} G_{R}\right)(t)\right)$

$L_{4}(t)=U(t)\left(\left(I^{1} K_{R}\right) I(t)-\frac{2}{3}\left(I^{1} G_{R}\right)(t)\right)$

and $\left(I^{1} K_{R}\right)(t)$ and $\left(I^{1} G_{R}\right)(t)$ are the first integrals of Eqs. (30a) and (30b) and are analogous to Eqs. (27a) and (27b) The analytical solution is compared with the computer simulation in Fig. 8, demonstrating that the computational result accurately reproduces the analytical solution.

Simulation of the relaxation test has been performed also for the case $\alpha \neq \beta$. Fig. 9 shows the comparison between analytical and FE solution for $\alpha=0.5$ and varying $\beta=0,0.25,0.5,0.75,1$ for both longitudinal and transverse stress. Fig. 10 shows comparison in the case of $\beta=0.5$ and $\alpha=0,0.25,0.5,0.75,1$. Both figures show the accuracy of the FE solutions, furthermore it is possible to appreciate that varying the values of the parameters $\alpha$ and $\beta$ leads to very different responses. In particular it is possible to note that when $\beta \neq \alpha$ the longitudinal stress is only slightly affected, while the transverse stress can change radically; when $\alpha>\beta$ the behaviour of the transverse stress can be even opposite to what one can expect intuitively: indeed in Fig. 9 the transverse stress increases instead of descreasing for $\beta=0$ (or for values of $\beta$ close to 0 ); in Fig. 10 instead, for $\alpha=1$ the transverse stress is even non-monotonic. This suggests that a very wide range of threedimensional behaviour by using different combinations of the fractional models and different values of the mechanical parameters, in particular by varying the orders $\alpha$ and $\beta$ a very wide range of time varying Poisson's ratios can be obtained [1,32,39].

\subsection{Euler-Bernoulli beam with FKV model}

In this section a benchmark problem with a fractional viscoelastic Euler-Bernoulli beam is shown; the material model is the FKV. The Euler Bernoulli beam model has been choosen because analytical solution [21] can be evaluated for the creep test. A 2D plain strain model of a viscoelastic Euler-Bernoulli beam under a uniformly distributed load which is ramped up to a constant value of $100 \mathrm{~N} / \mathrm{m}$ over $1 \mathrm{~s}$ is shown in Fig. 11. The beam is $5 \mathrm{~m}$ long, has a rectangular cross section with base $10 \mathrm{~cm}$ and height $20 \mathrm{~cm}$. The material has the following mechanical properties: $K_{\beta}=5 \times 10^{8} \mathrm{~Pa} \mathrm{~s}^{\beta}, K=10^{9} \mathrm{~Pa}, G_{\alpha}=3.75 \times 10^{8} \mathrm{~Pa} \mathrm{~s}^{\alpha}$, $G=7.5 \times 10^{8} \mathrm{~Pa}$, where $K$ and $G$ are the long term bulk and shear moduli, respectively, while $K_{\beta}$ and $G_{\alpha}$ are the viscoelastic bulk and shear coefficients, respectively; different values of $\alpha=\beta$ have been considered: $0,0.25,0.5,0.75,1$; the beam is modelled as simply supported. Fig. 11 shows the FE model of the beam, which consist of 100 $(10 \times 10 \mathrm{~cm}$ square $)$ finite elements. Points lying in the same vertical plane have been constrained to have the same vertical ( $y$-direction) dis-

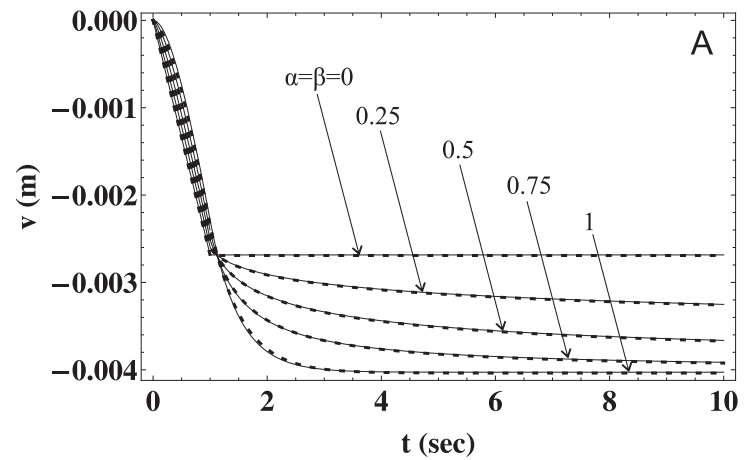

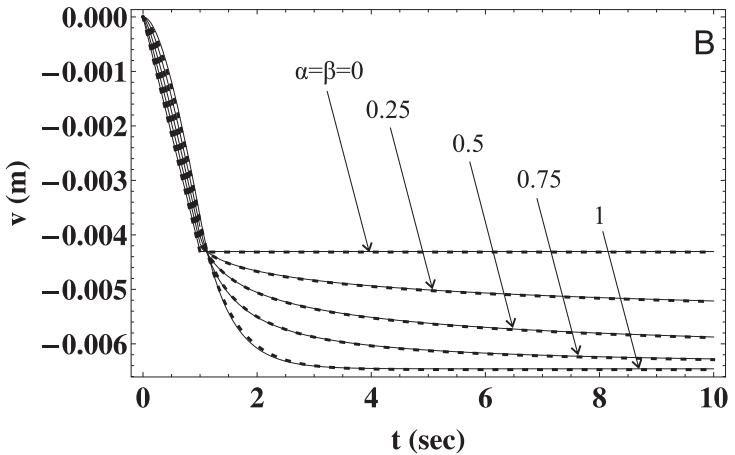

Fig. 12. Comparison between analytical and FEM results for displacements of two points of the beam. 


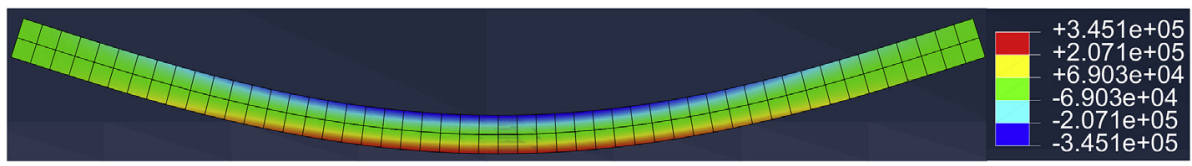

Fig. 13. Contour plot of longitudinal normal stress in the deformed configuration of the beam. Values of stress in Pa.

placements, in order to avoid vertical strain in the FE model that are not included in the analytical model. Displacements of the beam were monitored at $1 \mathrm{~m} \mathrm{(A)}$ and $2 \mathrm{~m}$ (B) from the left end of the beam. FEM results are compared with analytical results evaluated using the same approach as described in Ref. [21] in Fig. 12; the analytical solution is evaluated as described in the following.

Using concepts highlighted in Ref. [21], namely the correspondence principle [40] proved to be valid also for fractional viscoelasticity, it can be stated that the shape of the deformed configuration of the beam is the same as that for an elastic material and it only scales with time. Analytically, this corresponds to writing the time evolution of displacements of the beam simply by multiplying displacements given by the elastic solution by a time varying function, corresponding to the creep function of the mechanical model adopted, in this case a fractional Kelvin-Voigt model. The deformed configuration of a simply supported elastic Euler-Bernoulli beam with $x$ axis along its length and subjected to a uniform load $q$ in the $y$ direction is

$v_{e}(x)=\frac{q}{E I_{z}}\left(\frac{x^{4}}{24}-\frac{L x^{3}}{12}+\frac{L^{3} x}{24}\right)$

where $L$ is the length of the beam, $I_{z}$ is the second moment of area about the $z$ axis and $E$ is the Young's modulus. Then considering that the creep function of the fractional Kelvin-Voigt model reported in Table 2 the displacement at any point of the beam and at any time can be evaluated

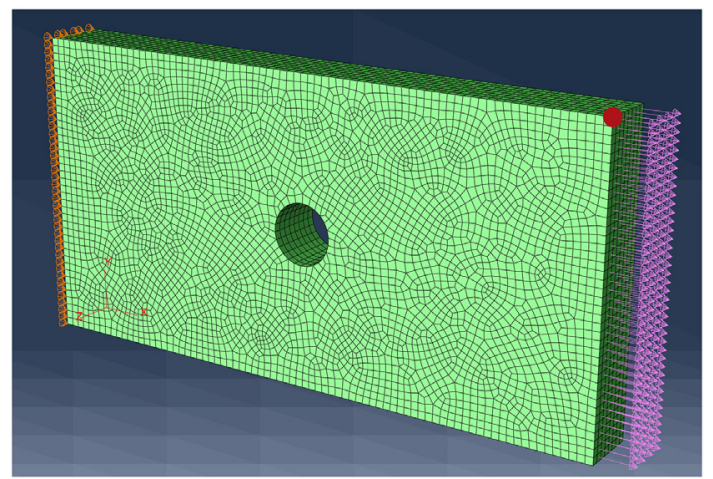

Fig. 14. Model of a plate with a hole. The red point has been used to generate Fig. 16 . (For interpretation of the references to colour in this figure legend, the reader is referred to the Web version of this article.)

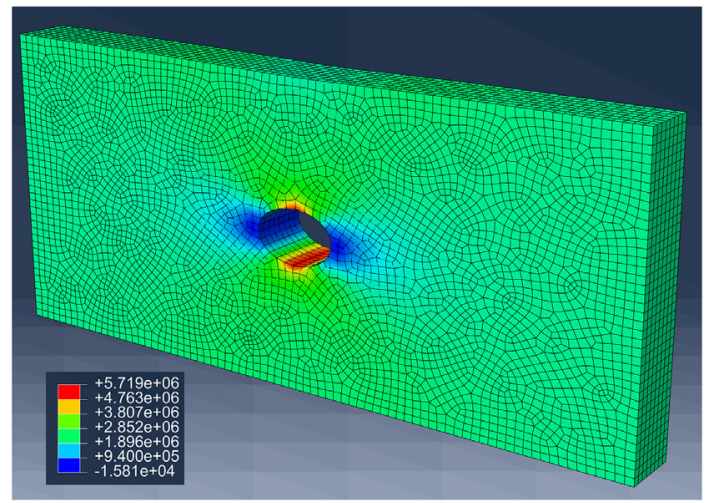

Fig. 15. Countour plot of $\sigma_{x x}$ stress. Values of stresses in Pa. as:

$v(x, t)=v_{e}(x)\left(L_{5}(t)-L_{5}\left(t-t_{0}\right)\right)$

where

$L_{5}(t)=t U(t)\left[1-E_{\beta, 2}\left(-\frac{E}{C_{\beta}} t^{\beta}\right)\right]$

is the first integral of the creep function of the FKV model. As in the test of the cube, analytical and FEM results are in very good agreement; a contour plot of $\sigma_{11}$ (stress in $x$-direction) is plotted in the deformed configuration in Fig. 13. The tests described above have been repeated for all of the models and also for oscillating and linear input (as done in one dimensional conditions in Section 5), both in quasi-static and in dynamic conditions. The structural models used are very simple and it has been possible to evaluate the analytical solutions for comparison. However, the importance of the routines presented in this work become more clear if models with complex geometry are analyzed. In the following section we show numerical results related to a plate with a hole made of a viscoelastic material whose behaviour is modelled through a range of fractional viscoelastic material models discussed in Section 4.

\section{A plate with a hole with different fractional viscoelastic models}

Here we analyze a 3D model of a plate with a hole shown in Fig. 14. This numerical example is useful in order to test the capability and the efficiency of the user subroutines to model the response of a large FE model. Furthermore, it is possible to compare results obtained by using different fractional viscoelastic models. The plate has dimensions $10 \times 5 \times 1 \mathrm{~cm}$, the hole is centered in the intersection between the diagonals of the $10 \times 5 \mathrm{~cm}$ faces and has a diameter of $1 \mathrm{~cm}$. A constant normal stress of $2 \mathrm{MPa}$ has been applied to one face of the plate as shown in Fig. 14. The stress is held constant for $10 \mathrm{~s}$, while the opposite face has been restrained in the normal direction. The model consists of 30648 3D brick linear elements with 8 nodes and 8 integration points. The material properties used for these analyses are reported in Section 6.1 for the FSLS1 model and in Section 6.2 for the Kelvin-Voigt, Maxwell and springpot model. In the springpot model the elastic moduli $K$ and $G$ are equal to zero. For each model we assume that $\alpha=\beta=0.3$. The constant time increment has been chosen as $\Delta t=0.1 \mathrm{~s}$. The analysis with a fractional Maxwell model was used to provide some information about:(1) the RAM memory usage during the analysis which was about 4 GBs and (2) the results file (.odb), which contains all the stress and

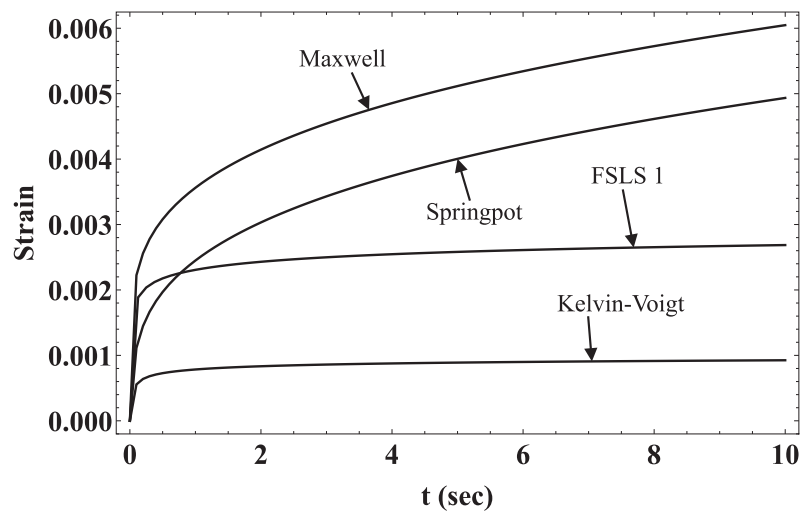

Fig. 16. Time evolution of $\varepsilon_{x x}$ for the red point in Fig. 14 and for different models. 
strain history for each integration point and all displacements at each node, which is about $1.2 \mathrm{GBs}$. The computational time is about $12 \mathrm{~min}$ on a single processor workstation.

We now compare the results for the different fractional viscoelastic models. First of all, we observe that, as expected, the distribution and the values of stresses obtained are the same for all models. This is due to the fact that the loading and boundary conditions are the same and equilibrium is satisfied by the same equations for all the models. A contour plot of $\sigma_{x x}$ is reported in Fig. 15 and is valid for all four models of Table 1. Furthermore, the distribution of strains within the plate is the same for all the constitutive models and it is analogous to the elastic case. Obviously, the evolution of the strains pattern with time is different for each of the viscoelastic models used. A comparison of $\varepsilon_{x x}$ for the red point in Fig. 14 for the different viscoelastic models is reported in Fig. 16. These two observations are in agreement with the correspondence principles ([40]). From Fig. 16 it can be observed that we can produce different time-dependent responses of the component (plate with the hole) by using the range of fractional viscoelastic models shown in Table 1. The choice of the appropriate material model needs to be guided by analyzing the data from creep or relaxation tests at the material level.

\section{Conclusions}

In this paper the implementation of a range of isotropic 3D fractional viscoelastic constitutive laws in a finite element context has been presented. We have implemented a series of 3D fractional viscoelastic models as user material subroutines in the FE commercial software Abaqus 6.14. The routines are suitable for both implicit and explicit integration schemes. We have shown that these models can be successfully implemented in finite element software by using the discretized version of the fractional derivative provided by Grünwald-Letnikov. We have also suggested a procedure to access the stress/strain history during the calculation process which is an essential requirement for implementing this class of models. The need to store the history of all strain and/or stress components can potentially lead to large amount of memory during the simulation; this problem has been investigated and two possible strategies have been discussed. Finally, a number of benchmark problems, for which the analytical solution is known, have been analyzed and the accuracy of the routines have been proved to very satisfying. The work presented here shows that it is possible to implement, in an easy and efficient manner, 3D fractional viscoelastic models into finite element software; it is shown that the implementation is straightforward also for researchers not familiar with fractional viscoelasticity and fractional calculus. The routines presented here are essential in order to model and study the behaviour of complex components made of fractional viscoelastic materials. The use of fractional viscoelasticity in conjunction with FE software extends the possibility to study, test and design viscoelastic components.

\section{Acknowledgments}

M. Di Paola would like to acknowledge the financial support of PRIN 2015 (under grant 2015JW9NJT_014, project "Advanced mechanical modeling of new materials and structures for the solution of 2020 Horizon challenges").

\section{Appendix A. Supplementary data}

Supplementary data related to this article can be found at https:// doi.org/10.1016/j.finel.2018.04.003.

\section{References}

[1] G. Alotta, O. Barrera, A.C.F. Cocks, M. Di Paola, On the behavior of a three-dimensional fractional viscoelastic constitutive model, Meccanica 52 (9) (2017) 2127-2142, https://doi.org/10.1007/s11012-016-0550-8.
[2] P.G. Nutting, A new general law of deformation, J. Franklin Inst. 191 (1921) $679-685$.

[3] R.L. Bagley, P.J. Torvik, On the appearance of the fractional derivative in the behavior of real materials, J. Appl. Mech. 51 (1984) 294-298.

[4] L. Deseri, M. Di Paola, M. Zingales, P. Pollaci, Power-law hereditariness of hierarchical fractal bones, Int. J. Num. Meth. Biomed. Eng. 29 (12) (2013) 1338-1360.

[5] G. Di Mino, G. Airey, M. Di Paola, F.P. Pinnola, G. D’Angelo, D. Lo Presti, Linear and non linear fractional hereditary constitutive laws of asphalt mixture, J. Civ. Eng. Manag. (2013), https://doi.org/10.3846/13923730.2014.914104.

[6] G. Alotta, M. Di Paola, A. Pirrotta, Fractional Tajimi-Kanai model for simulating earthquake ground motion, Bull. Earthq. Eng. 12 (2014) 2495-2506.

[7] I. Podlubny, Fractional Differential Equation, Academic Press, San Diego, 1999.

[8] G.S. Samko, A.A. Kilbas, O.I. Marichev, Fractional Integrals and Derivatives, Gordon and Breach Science, Amsterdam, 1993.

[9] M. Di Paola, M. Zingales, Exact machanical models of fractional hereditary materials, J. Rheol. 56 (2012) 983-1004.

[10] G.L. Slonimsky, On the law of deformation of highly elastic polymeric bodies, Dokl. Akad. Nauk SSSR 140 (2) (1961) 343-346.

[11] H. Schiessel, A. Blumen, Hierarchical analogues to fractional relaxation equations, J. Phys. Math. Gen. 26 (1993) 5057-5069.

[12] H. Schiessel, R. Metzeler, A. Blumen, T.F. Nonnemacher, Generalized viscoelastic models: their fractional equations with solutions, J. Phys. Math. Gen. 28 (1995) 6567-6584.

[13] R.L. Bagley, P.J. Torvik, Fractional Calculus - a different approach to the analysis of viscoelastically damped structures, AIAA J. 21 (5) (1983) 741-748.

[14] R.L. Bagley, P.J. Torvik, On the fractional calculus model of viscoelastic behavior, J. Rheol. 30 (1) (1986) 133-155.

[15] F.P. Pinnola, Statistical correlation of fractional oscillator response by complex spectral moments and state variable expansion, Commun. Nonlinear Sci. Numer. Simulat. 37 (2016) 14-30.

[16] N. Colinas-Armijo, M. Di Paola, F.P. Pinnola, Fractional characteristic times and dissipated energy in fractional linear viscoelasticity, Commun. Nonlinear Sci. Numer. Simulat. 39 (2016) 343-359.

[17] D.O. Craiem, F.J. Rojo, J.M. Atienza, G.V. Guinea, R.L. Armentano, Fractional calculus applied to model arterial viscoelasticity, Lat. Am. Appl. Res. 38 (2008) $141-145$.

[18] M. Di Paola, V. Fiore, F.P. Pinnola, A. Valenza, On the influence of the initial ramp for a correct definition of the parameters of fractional viscoelastic materials, Mech. Mater. 69 (1) (2014) 63-70.

[19] R.M. Guedes, A viscoelastic model for a biomedical ultra-high molecular weight polyethylene using the time-temperature superposition principle, Polym. Test. 30 (2011) 294-302.

[20] Y. Kobayashi, A. Kato, H. Watanabe, T. Hoshi, K. Kawamura, M.G. Fujie, Modeling of viscoelastic and nonlinear material properties of liver tissue using fractional calculations, J. Biomech. Sci. Eng. 7 (2) (2012) 117-187.

[21] M. Di Paola, R. Heuer, A. Pirrotta, Fractional visco-elastic Euler-Bernoulli beam, Int. J. Solid Struct. 50 (2013) 3505-3510.

[22] G. Alotta, G. Failla, M. Zingales, Finite element formulation of a non-local hereditary fractional order Timoshenko beam, J. Eng. Mech. ASCE (2015), https:// doi.org/10.1061/(ASCE)EM.1943-7889.0001035.

[23] G. Alotta, G. Failla, F.P. Pinnola, Stochastic analysis of a non-local fractional viscoelastic bar forced by Gaussian white noise, ASCE-ASME J. Risk Uncertain. Eng. Syst. Part B Mech. Eng. 3 (3) (2017) 030904, https://doi.org/10.1115/1. 4036702.

[24] S. Di Lorenzo, F.P. Pinnola, A. Pirrotta, On the dynamics of fractional visco-elastic beams, in: ASME 2012 International Mechanical Engineering Congress and Exposition, Volume 4: Dynamics Control and Uncertainty Parts A and B, 2012 4:9-15, https://doi.org/10.1115/IMECE2012-86566.

[25] Alotta G, Di Paola M, Failla G, Pinnola FP. On the dynamics of non-local fractional viscoelastic beams under stochastic agencies. Composites Part B; https://doi.org/ 10.1016/j.compositesb.2017.10.014.

[26] G. Alotta, N. Colinas-Armijo, Analysis of fractional viscoelastic material with mechanical parameters dependent on random temperature, ASCE-ASME J. Risk Uncertain. Eng. Syst. Part B Mech. Eng. 3 (3) (2017) 030906, https://doi.org/10. 1115/1.4036704.

[27] D. Badagliacco, N. Colinas-Armijo, M. Di Paola, A. Valenza, Evaluation of the temperature effect on the fractional linear viscoelastic model for an epoxy resin, in: AIP Conference Proceedings, vol. 1736, 2016, p. 020089, https://doi.org/10. 1063/1.4949664.

[28] N. Demirci, E. Tonuk, Non-integer viscoelastic constitutive law to model soft biological tissues to in-vivo indentation, Acta Bioeng. Biomech. 16 (4) (2014) $13-21$.

[29] A.D. Freed, K. Diethelm, Fractional calculus in biomechanics: a 3D viscoelastic model using regularized fractional derivative kernels with application to the human calcaneal fat pad, Biomechanics Model. Mechanobiol. 5 (2006) 203-215.

[30] M. Fukunaga, N. Shimizu, Fractional derivative constitutive models for finite deformation of viscoelastic materials, J. Comput. Nonlinear Dynam. 10 (6) (2015) 10:061002.

[31] H.H. Hilton, Generalized fractional derivative anisotropic viscoelastic characterization, Materials 5 (2012) 169-191.

[32] N. Makris, Three-dimensional constitutive viscoelastic laws with fractional order time derivatives, J. Rheol. 41 (1997) 1007-1020.

[33] M. Enelund, L. Mahler, K. Runesson, B.L. Josefson, Formulation and integration of the standard linear viscoelastic solid with fractional order rate laws, Int. J. Solid Struct. 36 (1999) 2417-2442. 
[34] S.A. Chester, C.V. Di Leo, L. Anand, A finite element implementation of a coupled diffusion-deformation theory for elastomeric gels, Int. J. Solid Struct. 52 (2015) $1-18$.

[35] O. Barrera, E. Tarleton, H.V. Tang, A.C.F. Cocks, Modelling the coupling between hydrogen diffusion and the mechanical behaviour of metals, Comput. Mater. Sci. 122 (2016) 219-228.

[36] K. Park, G.H. Paulino, Computational implementation of the PPR potential-based cohesive model in ABAQUS: educational perspective, Eng. Fract. Mech. 93 (2012) 239-262.

[37] G.W. Scott Blair, J.E. Caffyn, An application of the theory of quasi-properties to the treatment of anomalous strain-stress relations, Philos. Mag. A 40 (300) (1949) 80-94.
[38] ABAQUS 6.14, Providence, RI.

[39] R.S. Lakes, The time-dependent Poisson's ratio of viscoelastic materials can increase or decrease, Cell. Polym. 11 (1992) 466-469.

[40] W. Flugge, Viscoelasticity, Blaisdell Publishing Company, Massachusetts, 1967.

[41] E. Pegg, G. Alotta, O. Barrera, Elasto-plastic material models introduce error in finite element polyethylene wear predictions, in: European Orthopaedic Research Society Meeting, 14th-16th September 2016, Istituto Ortopedico Rizzoli, Bologna, Italy, 2016.

[42] G. Alotta, O. Barrera, E. Pegg, Viscoelastic material models for more accurate polyethylene wear estimation, Article in press, J. Strain Anal. Eng. Des. (2018). 